\title{
Nanoscale films covalently attached to conducting substrates: structure and dynamic behaviour of the layers
}

\author{
Alison J. Downard* \\ MacDiarmid Institute for Advanced Materials and Nanotechnology, \\ Department of Chemistry, University of Canterbury, Private Bag 4800, \\ Christchurch, New Zealand \\ Fax:+6433642110Ｅ-mail: Alison.downard@canterbury.ac.nz \\ ${ }^{*}$ Corresponding author
}

\begin{abstract}
Nanoscale organic films, typically 1-5 nm thick, can be grafted to conducting substrates by the reduction of aryldiazonium salts. The grafting procedure results in covalent attachment of the film to the substrate, through a direct bond between the aryl group and a surface atom. The strength of this bond depends on the substrate: for carbon substrates, a high bond energy gives extremely stable layers. This article describes our detailed electrochemical and atomic force microscopy studies of these films, which demonstrate that multilayer films do not incorporate close-packed layers of aryl groups. A loosely packed structure is consistent with the mechanism of the surface attachment process, which proceeds via the formation of aryl radicals. It is also consistent with the proposed route for multilayer formation which yields branching film growth. Our studies of the films have revealed that they can respond in an, at least partially, reversible manner to their environment, undergoing changes in thickness, barrier properties and wettability as they are exposed to different solvents and/or applied potentials. These switchable properties are proposed to originate in film swelling and shrinking which are possible because of the loosely packed multilayer structure.
\end{abstract}

Keywords: aryldiazonium salt; surface concentration; film thickness; density; redox probes; wettability; switchable properties

Reference for publisher use only

Biographical notes: Alison Downard received her $\mathrm{BSc}(\mathrm{Hons})$ degree and $\mathrm{PhD}$ from the University of Otago, New Zealand. Her PhD thesis concerned the synthesis and electrochemistry of tricobalt carbonyl clusters, under the supervision of Professors Brian Robinson and Jim Simpson. Alison then spent a postdoctoral year working on the nucleation and growth of conducting polymers with Professor Derek Pletcher at the University of Southampton. She follwed this with a two-year postdoctoral position with Professor T. J. Meyer at UNC-Chapel Hill where her research concerned electropolymerized multilayer and copolymeric structures based on substituted pyrroles. Since joining the Chemistry Department of the University of Canterbury in 1988, Alison has worked on various aspects of electrochemistry and electroanalysis, and for the past five years her research has focussed on nanoscale organic films covalently grafted to conducting substrates. 


\section{Introduction}

Surface modification aims to give new properties to a surface while maintaining the bulk properties of the material. Biocompatibility, wettability, adhesion, resistance to corrosion and wear-and-tear, and chemical functionality are a few examples of important surface properties which can be modulated by surface coatings. Methods for attachment of thin (sub-50 nm) organic layers to conducting substrates via covalent bonds directly between the modifier and surface atoms of the bulk material are receiving increasing attention [1; 2]. The stability of the covalent attachment and the simplicity of the procedures are attractive features of these methods. Most commonly, the reactions are initiated electrochemically, but can also occur spontaneously at room temperature, or with thermal or photolytic activation [3]. The first example of electrochemically-assisted covalent modification of graphitic carbon substrates was reported in 1990 [4]. Since then many studies have explored this general approach to grafting at carbon, metal and semiconductor substrates. In the electrochemical method, a radical is generated in solution at the electrode (substrate) surface by reduction or oxidation of the modifier. The radical appears to couple to the surface with formation of a covalent bond $[1 ; 2]$. The most studied reaction is reduction of an aryldiazonium cation to yield an aryl radical which binds to the surface. Electroreduction of iodonium salts leading to grafting of phenyl and alkynyl groups [5;6], reduction of alkenes resulting in thin polymeric layers [7], and oxidation of primary amines [4; 8-11] and arylacetates [12; 13] giving amine and methylene-based radicals respectively, are alternative approaches to surface grafting.

Much of our work in this area has focussed on grafting of 1-5 nm layers of aryl groups to graphitic carbon substrates via the electroreduction of aryldiazonium salts. Figure 1 shows a scheme, based on one recently proposed by Pinson and co-workers [14], which accounts for the formation of multilayered surface attached films from diazonium salts. Pathway B of the scheme shows formation of a C-C bonded multilayer structure. In pathway $\mathrm{C}$ an alternative route to a multilayer film is outlined, based on reaction between a diazonium cation and a cyclohexadienyl radical. This pathway, which is proposed to operate concurrently with pathway $\mathrm{B}$, explains the spectroscopic detection of azo groups within the films [14-17]. The Scheme is depicted for a generalised substrate; when the substrate is carbon, aryl groups graft to the surface via $\mathrm{C}-\mathrm{C}$ bonds, giving layers that largely resist desorption when sonicated in aggressive solvents, heated under vacuum to at least $400{ }^{\circ} \mathrm{C}$ [1] and treated to potential cycling over a wide potential range [15]. These properties suggest that when stability of the surface attachment is of prime importance, grafting onto carbon substrates from aryldiazonium salt solutions should be considered as a method of choice.

In addition to the stability of grafted layers, most graphitic carbons have the advantages of ease-of-handling, low cost, high mechanical stability and good electrical conductivity. We have used glassy carbon (GC) and pyrolysed photoresist films (PPFs) for our studies. GC is a very popular, commercially available, electrode material which can be readily polished to an electrochemically active and reproducible state [18]. PPF is prepared in the laboratory and has similar properties to GC but has a much smoother surface, with typical average surface roughness less than $0.5 \mathrm{~nm}$ [19-21]. This molecular level roughness makes PPF a useful material for imaging molecular layers using atomic force microscopy (AFM). 
Figure 1 Proposed scheme for formation of multilayer films by reduction of aryldiazonium salts. Adapted from reference 14. Reaction A shows that, once initiated, film growth can be sustained without the introduction of additional radicals. Pathway $\mathrm{B}$ is the route to a totally $\mathrm{C}-\mathrm{C}$ bonded multilayer. Pathways $\mathrm{C}$ shows attack of an aryldiazonium cation on a grafted cyclohexadienyl radical resulting in incorporation of azo linkages in the film.
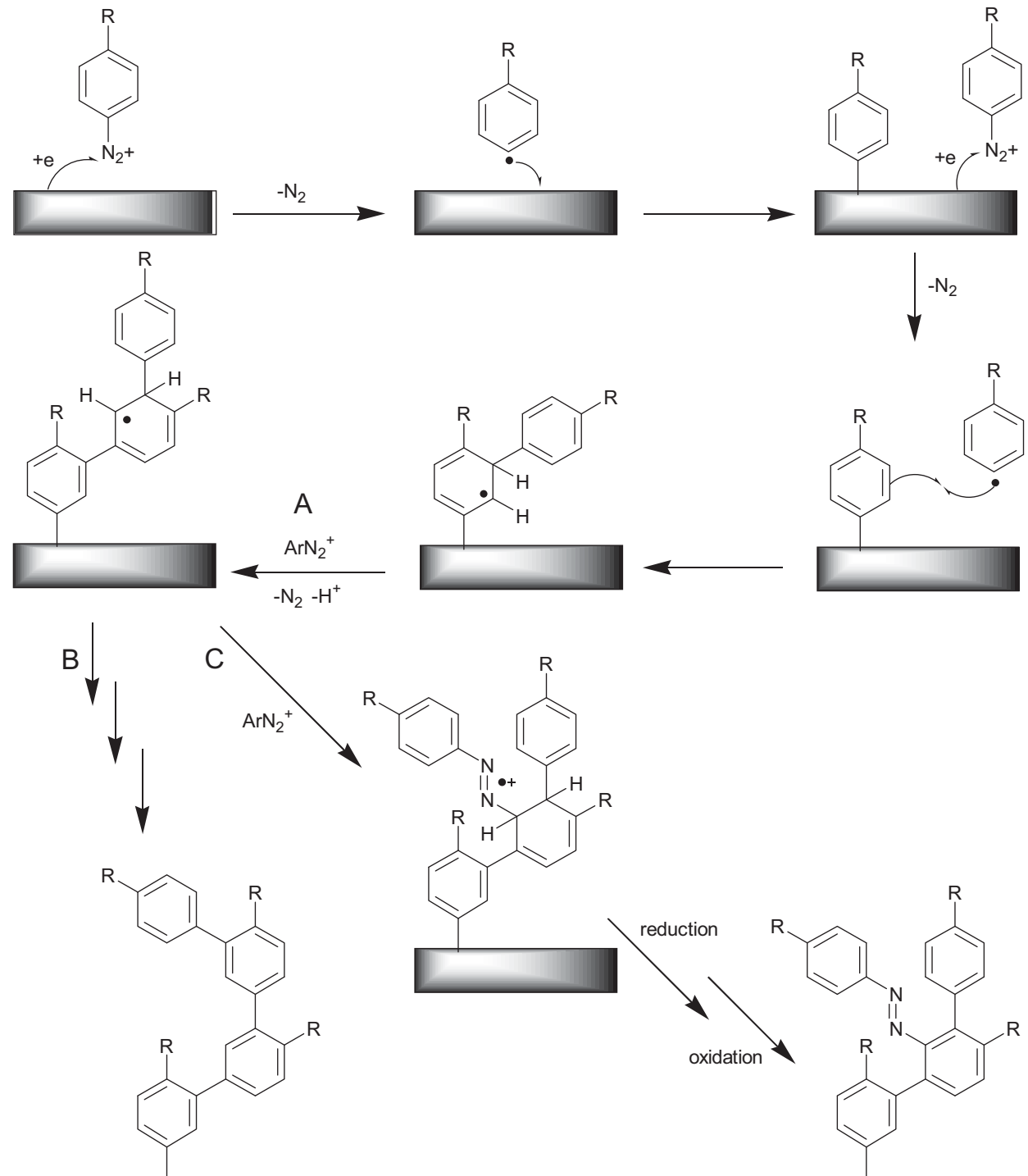

One of the goals of our studies of electrografted films on graphitic carbon, and recently gold, substrates is to gain a better understanding of film structure, properties and reactivity. In this article, the results from a number of investigations are discussed, and our current understanding of the structure and properties of films grafted by reduction of aryldiazonium salts is presented. These nanoscale, covalently attached, coatings appear to be a promising route to surfaces with switchable properties. 


\section{Film packing}

A close-packed layer of phenyl groups on an ideal planar surface has a calculated surface concentration of $12-13 \times 10^{-12} \mathrm{~mol} \mathrm{~cm}^{-2}[22 ; 23]$. This value is frequently quoted when making quantitative evaluations of films grafted by the reduction of aryldiazonium salts. However, for multilayer films, at least, the film growth mechanism shown in Figure 1 must preclude the formation of close-packed layers of phenyl groups. A branching film growth seems inevitable for para substituted phenyl modifiers, and it is not reasonable to propose that the resultant branched polyphenylene oligomers incorporate phenyl groups in a close-packed arrangement. The situation is less clear for the layer of aryl groups directly attached to the surface. Radical reactions are difficult to control and the strong bonds to the substrate and within the layers are assumed to prevent reorganization of the initially-formed layers. In other words, once a radical has reacted with the surface or an already grafted group, it is fixed in position. This is in contrast to the self assembly of alkanethiols on gold surfaces which, over time, produces an increasingly organized layer. Examination of Figure 1 makes it clear that once a phenyl group has coupled with a group already grafted to the surface, it effectively blocks access to the underlying surface. This would favour reaction of subsequently produced radicals with the film, rather than the substrate. Another factor possibly preventing close packing on GC and PPF is the inhomogeneity of the surfaces which incorporate both $\mathrm{sp}^{2}$ and $\mathrm{sp}^{3} \mathrm{C}$, basal plane and edge plane graphite sites and, for the latter, a mixture of oxygen functionalities, H-termination and radicals or other reactive species [18-21]. The mechanism for reaction of aryl radicals with these surfaces is not known however reaction at the basal plane has been shown to be much slower than at the edge plane [22; 24]. The relative rates of reaction at different edge plane sites is also not known, neither is the relative rate of reaction between aryl radicals and surface sites $v s$ aryl radicals and already grafted aryl groups. These (relative) reaction rates are likely to depend on the particular aryldiazonium derivative, and possibly on conditions used during grafting. In addition to reaction rate issues, the extent to which surface inhomogeneity determines the packing of the first layer of grafted groups must depend on the scale of the inhomogeneity relative to the molecular size of the modifier.

To address the question of film packing, we have used a combination of electrochemistry to determine the surface concentration of electroactive modifiers, and AFM depth profiling to determine the thickness of films. The depth profiling technique involves using an AFM tip to remove a section of film grafted to a PPF substrate, and then scanning across the scratch to determine its depth and hence the film thickness [25]. Figure 2a shows a cyclic voltammogram of a PPF surface with a film grafted from $p$ nitrobenzenediazonium salt [25]. In acid solution, the reduction of nitrophenyl groups at $E_{\mathrm{pc}} \approx-0.7 \mathrm{~V}$ affords the aminophenyl and hydroxylaminophenyl groups through the reactions described in equations 1 and 2 . A four-electron reduction of nitrophenyl yields the hydroxylaminophenyl group which may be further reduced to aminophenyl in a second two-electron step. The redox response observed at $E_{1 / 2} \approx 0.3 \mathrm{~V}$ is due to the hydroxylaminophenyl/nitrosophenyl couple (equation 3) which only appears after formation of hydroxylaminophenyl groups on the first reduction scan (equation 1).

$$
\begin{aligned}
& \text { surface-Ph-NO }+4 \mathrm{H}^{+}+4 \mathrm{e}^{-} \rightarrow \text { surface- } \mathrm{Ph}-\mathrm{NHOH}+\mathrm{H}_{2} \mathrm{O} \\
& \text { surface- } \mathrm{Ph}-\mathrm{NHOH}+2 \mathrm{H}^{+}+2 \mathrm{e}^{-} \rightarrow \text { surface }-\mathrm{Ph}-\mathrm{NH}_{2}+\mathrm{H}_{2} \mathrm{O} \\
& \\
& \text { PPF- } \mathrm{Ph}-\mathrm{NHOH} \leftrightarrow \text { PPF- } \mathrm{Ph}-\mathrm{NO}+2 \mathrm{H}^{+}+2 \mathrm{e}^{-}
\end{aligned}
$$


Figure 2 a) Cyclic voltammogram, first two scans, obtained in $0.1 \mathrm{M} \mathrm{H}_{2} \mathrm{SO}_{4}$ (scan rate $=200$ $\mathrm{mV} \mathrm{s}^{-1}$ ) for reduction of a nitrophenyl film on PPF; b) plot of electrochemically determined surface concentrations of nitrophenyl groups $v s$ thickness of as-prepared films determined by AFM depth profiling; c) molecular dimension of surface-attached nitrophenyl group. Figure adapted from reference 25 .
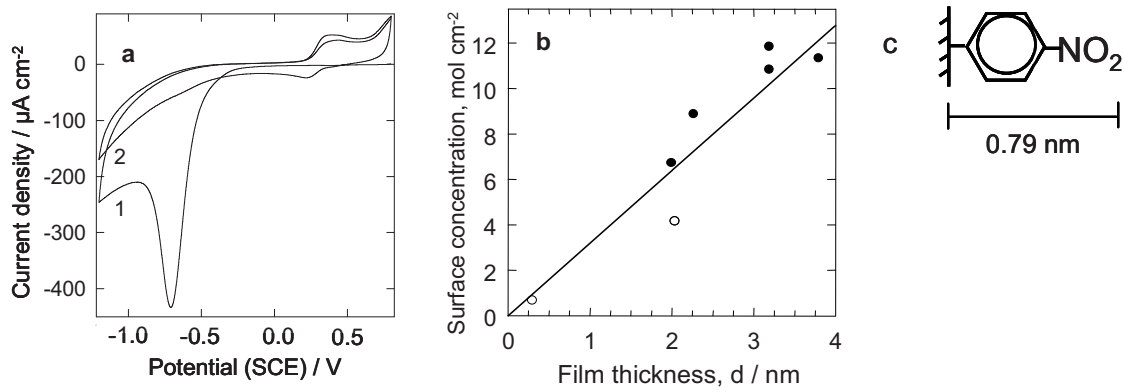

Determining the areas of the main reduction peak and small oxidation peak at $E_{\mathrm{pa}} \approx 0.4 \mathrm{~V}$ enables the charge associated with each redox process to be determined. From the charge, and taking the account of the stoichiometry of the reaction in equations 1-3, the total concentration of electroactive nitrophenyl groups in the film can be established. A series of nitrophenyl films were grafted to PPF and their surface concentrations measured by this method. The thicknesses of the same films, prior to reduction in acid, were measured by AFM depth profiling, giving the data shown in Figure $2 \mathrm{~b}$. The linear relationship between surface concentration and film thickness, and the zero intercept of the plot, suggest that the film grows with constant density and that all nitrophenyl groups are electrochemically accessible (and hence contributing to the measured charge). From the slope of the plot and length of the nitrophenyl modifier $(0.79 \mathrm{~nm}$, Figure $2 \mathrm{c})$, a surface concentration of $(2.5 \pm 0.5) \times 10^{-10} \mathrm{~mol} \mathrm{~cm}^{-2}$ nitrophenyl groups per monolayer equivalent (i.e. per slice of film with the thickness of a monolayer) was estimated. This calculation assumes that all nitrophenyl groups are oriented perpendicular to the surface and hence represents a maximum surface concentration per monolayer equivalent. This surface concentration is $(21 \pm 3) \%$ of an ideal close-packed monolayer and supports the notion that multilayer films do not, and cannot, have close packed phenyl layers [25].

As a result of studies undertaken since the above work, it now appears that the reported uncertainty in surface concentration may be an underestimate [17]. The $20 \%$ uncertainty quoted above, was based on the level of reproducibility of data and the error in the slope of the plot. All peak areas were measured using a consistently applied peak fitting procedure. However, it is now clear that establishing the 'correct' baseline to use when peak fitting is subject to significant uncertainty. During repeat potential scans, such as those shown in Figure 2a, the voltammetric 'baseline' changes continuously, due to both reduction of nitrophenyl groups and to changes in permeability of the film. This means that determining peak areas is an unavoidably uncertain method.

When the same electrochemical and AFM analysis decribed above for nitrophenyl films was applied to films grafted from $p$-nitroazobenzenediazonium salt, the surface concentration $v s$ film thickness plot shown in Figure 3a was obtained [26]. For this modifier, the relationship between electrochemically-determined surface concentration and film thickness is not linear and it appears that while film thickness increases from 4 to $6 \mathrm{~nm}$, the surface concentration remains constant at approximately $8.4 \times 10^{-10} \mathrm{~mol} \mathrm{~cm}^{-}$

${ }^{2}$. This is not physically reasonable and we can conclude that for this film, not all 
nitroazobenzene groups are electrochemically active, at least not in the $0.1 \mathrm{M} \mathrm{H}_{2} \mathrm{SO}_{4}$ medium used to determine the surface concentration. This finding highlights that deducing information about film packing density from electrochemical measurement of surface concentrations must be applied cautiously. Reliable information can only be obtained after confirming a linear relationship between surface concentration and film thickness. Single measurements of surface concentration cannot be assumed to give meaningful information.

Figure 3 a) Plot of electrochemically determined surface concentration of nitroazobenzene groups vs film thickness determined by AFM depth profiling; b) surface-attached nitroazobenzene group. Figure adapted from reference 26 .

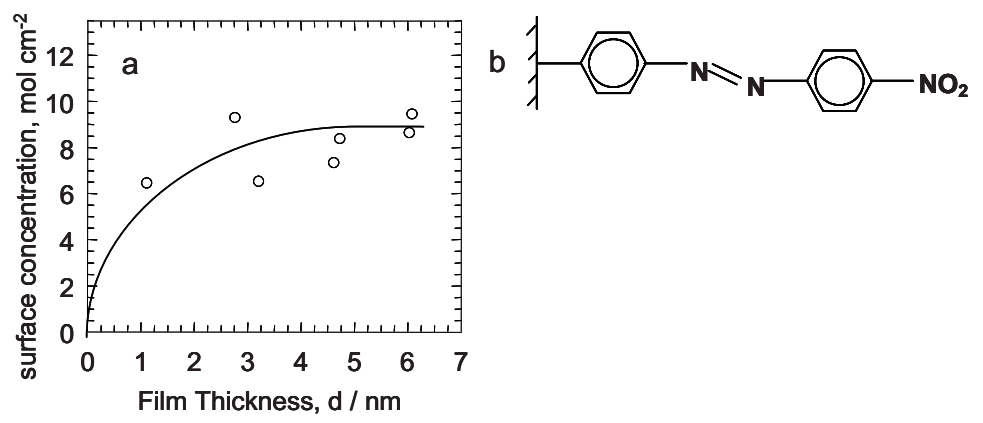

We have looked at the question of film packing from a different angle by grafting films to gold surfaces and monitoring the gold surface electrochemistry [27]. Gold surface atoms can be oxidised in aqueous media to gold oxide (or hydroxide) and subsequently reduced back to their initial state (Figure 4a). Measuring the decrease in charge associated with gold oxide reduction after film grafting, allows calculation of the fraction of gold surface atoms remaining 'free' and, by difference, the fraction involved in binding to the modifying layer. Quantitative analysis based on gold surface electrochemistry requires very careful preparation of the gold surface to ensure a reproducible state can be achieved before and after grafting. Under these conditions, we found that for grafted films of methylphenyl (Figure 4b) and carboxyphenyl (Figure 4c) groups, 20 - 30\% of gold surface atoms are non-electroactive after grafting and hence we deduce that these atoms are bound to the first layer of phenyl groups in the films. By taking account of the number of gold atoms at the surface and the relative sizes of gold atoms and phenyl groups, we estimate that the surface coverage of phenyl modifiers directly attached to the gold surface is $(3-4) \times 10^{-10} \mathrm{~mol} \mathrm{~cm}^{-2}$ [27]. Considering that the gold surface is rougher than PPF, this value is in excellent agreement with the value we obtained for nitrophenyl groups attached to PPF $\left((2.5 \pm 0.5) \times 10^{-10} \mathrm{~mol} \mathrm{~cm}^{-2}\right)$.

In summary, we obtain close agreement in our film surface concentration values obtained using different methods. Multilayer films appear to grow with constant density so that the concentration of the first layer is the same as subsequent layers. This concentration is in the region of $(2.5 \pm 0.5) \times 10^{-10} \mathrm{~mol} \mathrm{~cm}^{-2}$ for para substituted phenyl derivatives at a very smooth surface. Consistent with our reasoning that the branching growth of such films cannot lead to a close packed structure, the surface concentration corresponds to approximately $21 \%$ of an ideal close-packed monolayer of phenyl groups. 
Figure 4 a) Cyclic voltammogram of a gold electrode in $0.01 \mathrm{M} \mathrm{HClO}_{4}$ at $100 \mathrm{mV} \mathrm{s}^{-1}$, after cycling over the indicated potential range for $3 \mathrm{~h}$ at $50 \mathrm{mV} \mathrm{s}^{-1}$; b) methylphenyl surface modifier; c) carboxyphenyl surface modifier. Figure adapted from reference 27.

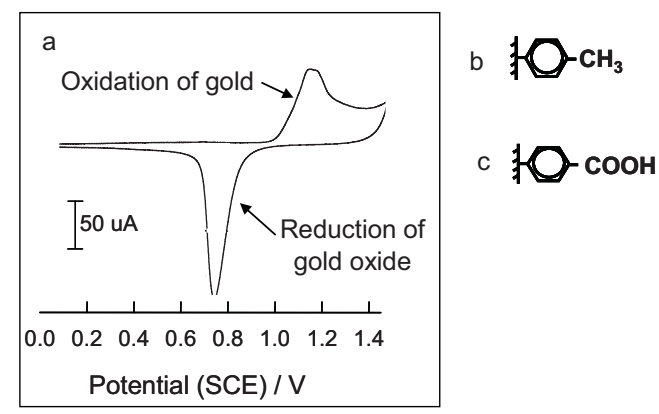

\section{Dynamic film behaviour}

During our quantitative investigations of film packing described above, and through other studies of film properties such as blocking behaviour of films towards redox probes in solution, we have started to gather evidence for some interesting dynamic behaviour of the films. We have observed that when treated with different solvents and applied potentials, films shrink and swell, become more or less blocking to solution redox probes and exhibit changes in wettability. These observations, which indicate that film properties can be switched by their environment, are not surprising, considering the loosely packed film structure.

Changes in average thickness as a result of applied potential (and possibly changes in solvent) are shown in Figure 5 for nitrophenyl and 1-naphthylmethyl films [13; 28]. Each data set corresponds to one film, for which AFM depth profiling measurements were undertaken before and after each treatment.

Figure 5 Changes in average film thickness following film treatments. The 1-naphthylmethyl film was grafted by oxidation of the corresponding carboxylate. Film treatments: 1 : as prepared film; 2: 2 potential cycles, 0.7 to $-1.3 \mathrm{~V}$ (SCE), $200 \mathrm{mV} \mathrm{s}^{-1}$, in $0.25 \mathrm{M} \mathrm{H}_{2} \mathrm{SO}_{4}$; 3: treated to film preparation conditions but without modifier; $4: 2$ potential cycles, 0 to $-2.9 \mathrm{~V}(\mathrm{Ag}), 20 \mathrm{~V} \mathrm{~s}^{-1}$, in $0.1 \mathrm{M}\left[\mathrm{Bu}_{4} \mathrm{~N}_{3} \mathrm{BF}_{4}\right.$-acetonitrile. The three sets of data for each film were obtained for the same film, with treatment order left to right.

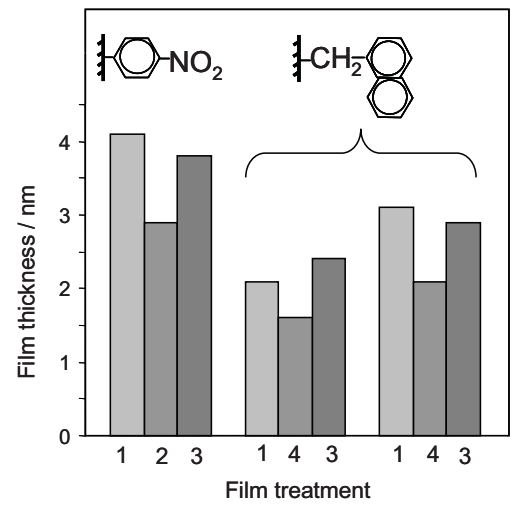


For all freshly-prepared films, the decrease in film thickness after cycling to negative potentials is significant (considering experimental uncertainties), however all films return to their initial thickness (within experimental uncertainty) after re-treating to the grafting conditions, but in the absence of modifier. The reversibility of the changes indicates that significant loss of film material does not occur during scans to negative potentials, or, for the nitrophenyl film, during reduction in acid. Hence the decrease in film thickness must be due to the film structure becoming more compact. A number of processes could contribute to film shrinking and swelling, such as potential-dependent incorporation or loss of solvent and ions, and the effect of electrode charge on film orientation. Although the details are as yet unknown, these findings highlight two important points: first, a decrease in film thickness does not necessarily indicate loss of film from the surface, and second, when deducing film density from surface concentration and film thickness data, the pre-treatment of the film will influence its thickness and hence the density.

The barrier properties of films grafted to electrode surfaces are commonly assessed by performing cyclic voltammetry of redox probe species at the bare and grafted electrodes. The presence of a grafted insulating layer can affect the voltammetry in several ways. For a compact and continuous multilayer film, the distance for electron transfer between the probe and the surface is increased by the intervening film, and hence the rate decreases [29]. Cyclic voltammograms at the grafted surface will be indicative of slow electron transfer. Films which are loosely packed may be somewhat porous to the redox probe allowing it to approach closer to the surface than is the case for a compact film. Hence for loosely packed films, the decrease in electron transfer rate compared from that at a bare surface may be significantly less than at a compact film. If the film has pinholes, the effect on redox probe voltammetry depends on the size and spacing of the pinholes [30]. Small, widely spaced pinholes will give rise to steady-state voltammetry as the pinholes function as individual micro- or nanoelectrodes. When the pinhole spacing is sufficiently small to allow overlap of the diffusion fields, the voltammetry will resemble that for a species with slow electron transfer.

In a study based on films grafted by the electroreduction of aryldiazonium salts, we have observed solvent-induced reversible changes in the blocking properties of the films to the ferricyanide redox probe. Methylphenyl and carboxyphenyl films (Figure 4b,c) were grafted to gold surfaces by reduction of the corresponding diazonium salt [27]. After an initial rinsing step, the carboxyphenyl-modified surface was sonicated alternately in water and petroleum ether for $20 \mathrm{~min}$ each, and the methylphenyl film in acetonitrile and water. After each sonication period, voltammograms were recorded of ferricyanide in phosphate buffer $/ 0.1 \mathrm{M} \mathrm{KCl}$. The voltammograms of ferricyanide in Figure 6a indicate that after sonication in water, the carboxyphenyl film is moderately blocking, but that when the film is then sonicated in petroleum ether and returned to the ferricyanide solution, it is significantly more blocking. After subsequent sonication in water, the voltammogram is essentially the same as after the first sonication in water and similarly for a further sonication in petroleum ether, i.e. the changes in barrier properties are reversible. Figure $6 \mathrm{~b}$ shows voltammograms recorded at the methylphenyl film after similar sonication steps. This film also shows reversible blocking properties switching from being moderately blocking after sonication in water to less blocking after sonication in acetonitrile. 
Figure 6 a,b) Cyclic voltammograms (scan rate $=100 \mathrm{mV} \mathrm{s}^{-1}$ ) of $0.5 \mathrm{mM}$ ferricyanide in phosphate buffer/0.1 M KCl, pH 7 at a) carboxyphenyl modified GC; b) methylphenylmodified GC. Scan 1 was obtained at polished electrode, scan 2 at the as-prepared modified electrode and scans 3 and 4 after 20 min sonication in the solvents indicated. Experiments were performed in the order 1-4. Sonication was performed at $0{ }^{\circ} \mathrm{C}$ for a, and $45^{\circ} \mathrm{C}$ for b. c) water contact angles on methylphenyl film after sonication (20 min) in the solvents indicated; experiments were performed in order left to right. Figure adapted from reference 27 .
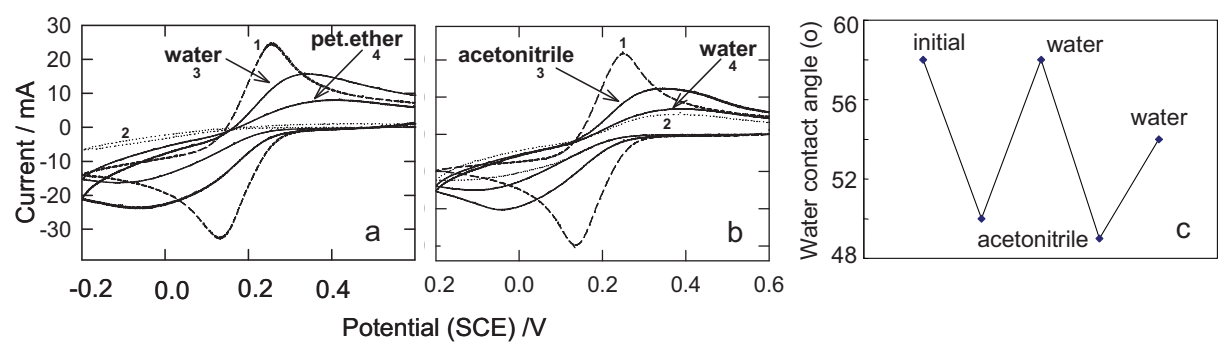

In the same study, the effects of sonication on surface wettability were monitored by measuring water contact angles of the films. Figure $6 \mathrm{c}$ shows the results for a methylphenyl film. Water contact angles are smaller after sonication in acetonitrile than in water, indicating a more hydrophilic surface after acetonitrile treatment. The changes in wettability show reasonable reversibility, although the differences in angles after sonication in the two solvents angles become progressively smaller. Qualitatively similar behaviour, but with the opposite wettability changes, was observed for carboxyphenyl films: the contact angles were smaller (indicating a more hydrophilic film) after sonication in water than in petroleum ether [27].

To account for the solvent-dependent film barrier properties and wettability, the model of film behaviour outlined in Figure 7 is proposed. The Figure shows that when the film is in a swollen state, the film porosity allows redox probe to closely approach the electrode surface and undergo electron transfer. When the film shrinks and is less porous, redox probe is largely confined to the outside of the film, where the large distance for electron transfer gives voltammetry consistent with a more blocking layer. After sonication in a 'like' solvent (water for carboxyphenyl, and acetonitrile for methylphenyl films) the film has the swollen structure depicted in a, whereas after sonication in an 'unlike' solvent (petroleum ether and water for carboxyphenyl and methylphenyl, respectively), the film structure is more compact as shown in b. Film wettability changes can also be understood by considering film structural changes. Both films are more hydrophilic after sonication in the like solvent which gives a more porous film structure. A swollen, porous structure allows the water droplet to wet the film more effectively, leading to a lower contact angle.

Figure 7 Proposed model for film structure in like and unlike solvents

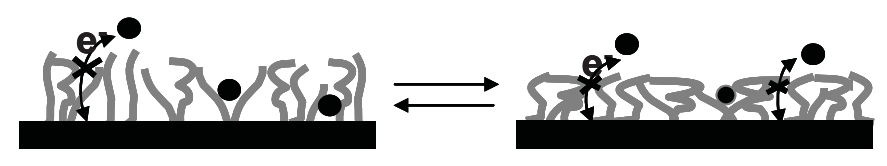

a) Like solvent

Film is swollen and porous b) Unlike solvent

Film is compact and less porous 


\section{Conclusions}

Nanoscale films covalently grafted to conducting substrates have interesting properties because they are not close-packed. The open, branching structure leads to significant free volume in the films, and allows as-prepared films to decrease their thickness by substantial amounts when the film environment is changed. Through careful AFM studies we have shown that thickness changes can be largely reversed by reversing the film environment. Changes in film thickness generate changes in the barrier properties of the films towards solution redox probes and changes in film wettability. Switchable properties are of great interest for the preparation of smart materials and the very stable covalent attachment and low thickness of these grafted films make them attractive for further development. In ongoing studies we aim to gain a better understanding of methods which can be used to induce changes in film thickness, and to identify other film properties that can be reversibly switched.

\section{Acknowledgements}

This work was supported by the MacDiarmid Institute for Advanced Materials and Nanotechnology and the University of Canterbury, Christchurch, New Zealand.

\section{References}

1 Downard, A.J. (2000) 'Electrochemically assisted covalent modification of carbon electrodes', Electroanalysis, Vol. 12, No. 14, pp. 1085-1096.

2 Pinson, J. and Podvorica, F. (2005) 'Attachment of organic layers to conductive or semiconductive surfaces by reduction of diazonium salts', Chem. Soc. Rev., Vol. 34, No. 5, pp. 429-439.

3 Barrière, F. and Downard, A.J. (2007) 'Covalent modification of graphitic carbon substrates by non-electrochemical methods', submitted to J. Solid State Electrochem.

4 Barbier, B., Pinson, J., Desarmot, G., and Sanchez, M. (1990) 'Electrochemical bonding of amines to carbon fiber surfaces toward improved carbon-epoxy composites', J. Electrochem. Soc., Vol. 137, No. 6, pp. 1757-1764.

5 Vase, K.H., Holm, A.H., Norrman, K., Pedersen, S.U., and Daasbjerg, K. (2007) 'Covalent grafting of glassy carbon electrodes with diaryliodonium salts: New aspects', Langmuir, Vol. 23, No. 7, pp. 3786-3793.

6 Vase, K.H., Holm, A.H., Pedersen, S.U., and Daasbjerg, K. (2005) 'Immobilization of aryl and alkynyl groups onto glassy carbon surfaces by electrochemical reduction of iodonium salts', Langmuir, Vol. 21, No. 18, pp. 8085-8089.

7 Palacin, S., Bureau, C., Charlier, J., Deniau, G., Mouanda, B., and Viel, P. (2004) 'Moleculeto-metal bonds: Electrografting polymers on conducting surfaces', Chemphyschem, Vol. 5, No. 10, pp. 1469-1481.

8 Adenier, A., Chehimi, M.M., Gallardo, I., Pinson, J., and Vila, N. (2004) 'Electrochemical oxidation of aliphatic amines and their attachment to carbon and metal surfaces', Langmuir, Vol. 20, No. 19, pp. 8243-8253.

9 Cruickshank, A.C., Tan, E.S.Q., Brooksby, P.A., and Downard, A.J. (2007) 'Are redox probes a useful indicator of film stability? An electrochemical, AFM and XPS study of electrografted amine films on carbon', Electrochem. Commun., Vol. 9, No. 7, pp. 1456-1462. 
10 Deinhammer, R.S., Ho, M., Anderegg, J.W., and Porter, M.D. (1994) 'Electrochemical oxidation of amine-containing compounds: A route to the surface modification of glassy carbon electrodes', Langmuir, Vol. 10, No. 4, pp. 1306-1313.

11 Downard, A.J., Tan, E.S.Q., and Yu, S.S.C. (2006) 'Controlled assembly of gold nanoparticles on carbon surfaces', New. J. Chem., Vol. 30, No. 9, pp. 1283-1288.

12 Andrieux, C.P., Gonzalez, F., and Saveant, J.M. (1997) 'Derivatization of carbon surfaces by anodic oxidation of arylacetates. Electrochemical manipulation of the grafted films', J. Am. Chem. Soc., Vol. 119, No. 18, pp. 4292-4300.

13 Brooksby, P.A., Downard, A.J., and Yu, S.S.C. (2005) 'Effect of applied potential on arylmethyl films oxidatively grafted to carbon surfaces', Langmuir, Vol. 21, No. 24, pp. 11304-11311.

14 Doppelt, P., Hallais, G., Pinson, J., Podvorica, F., and Verneyre, S. (2007) 'Surface modification of conducting substrates. Existence of azo bonds in the structure of organic layers obtained from diazonium salts', Chem. Mater., Vol. 19, No. 18, pp. 4570-4575.

15 D'Amours, M. and Belanger, D. (2003) 'Stability of substituted phenyl groups electrochemically grafted at carbon electrode surface', J. Phys. Chem. B, Vol. 107, No. 20, pp. 4811-4817.

16 Hurley, B.L. and McCreery, R.L. (2004) 'Covalent bonding of organic molecules to cu and al alloy 2024 t3 surfaces via diazonium ion reduction', J. Electrochem. Soc., Vol. 151, No. 5, pp. B252-B259.

17 Yu, S.S.C., Tan, E.S.Q., Jane, R.T., and Downard, A.J. (2007) 'An electrochemical and xps study of reduction of nitrophenyl films covalently grafted to planar carbon surfaces', Langmuir, Vol. 23, No. 22, pp. 11074-11082.

18 McCreery, R.L. (1991), in A. J. Bard (Ed.), Electroanalytical chemistry, Vol. 17, pp. 221-374, Dekker.

19 Kim, J., Song, X., Kinoshita, K., Madou, M., and White, B. (1998) 'Electrochemical studies of carbon films from pyrolyzed photoresist', J. Electrochem. Soc., Vol. 145, No. 7, pp. 23142319 .

20 Kostecki, R., Schnyder, B., Alliata, D., Song, X., Kinoshita, K., and Kotz, R. (2001) 'Surface studies of carbon films from pyrolyzed photoresist', Thin Solid Films, Vol. 396, No. 1,2, pp. 36-43.

21 Ranganathan, S. and McCreery, R.L. (2001) 'Electroanalytical performance of carbon films with near-atomic flatness', Anal. Chem., Vol. 73, No. 5, pp. 893-900.

22 Allongue, P., Delamar, M., Desbat, B., Fagebaume, O., Hitmi, R., Pinson, J., and Saveant, J.M. (1997) 'Covalent modification of carbon surfaces by aryl radicals generated from the electrochemical reduction of diazonium salts', J. Am. Chem. Soc., Vol. 119, No. 1, pp. 201207.

23 Liu, Y.-C. and McCreery, R.L. (1995) 'Reactions of organic monolayers on carbon surfaces observed with unenhanced raman spectroscopy', J. Am. Chem. Soc., Vol. 117, No. 45, pp. 11254-11259.

24 Ray, K., III and McCreery, R.L. (1997) 'Spatially resolved Raman spectroscopy of carbon electrode surfaces: Observations of structural and chemical heterogeneity', Anal. Chem., Vol. 69, No. 22, pp. 4680-4687.

25 Brooksby, P.A. and Downard, A.J. (2004) 'Electrochemical and atomic force microscopy study of carbon surface modification via diazonium reduction in aqueous and acetonitrile solutions', Langmuir, Vol. 20, No. 12, pp. 5038-5045.

26 Brooksby, P.A. and Downard, A.J. (2005) 'Multilayer nitroazobenzene films covalently attached to carbon. An AFM and electrochemical study', J. Phys. Chem. B, Vol. 109, No. 18, pp. 8791-8798.

27 Paulik, M.G., Brooksby, P.A., Abell, A.D., and Downard, A.J. (2007) 'Grafting aryl diazonium cations to polycrystalline gold: Insights into film structure using gold oxide 
reduction, redox probe electrochemistry, and contact angle behavior', J. Phys. Chem. C, Vol. 111, No. 21, pp. 7808-7815.

28 Yu, S.S.C. and Downard, A.J. (2005) 'Dynamic behavior of organic thin films attached to carbon surfaces', e- J. Surf. Sci. Nanotech., Vol. 3, pp. 294-298.

29 Miller, C., Cuendet, P., and Graetzel, M. (1991) 'Adsorbed omega-hydroxy thiol monolayers on gold electrodes: Evidence for electron tunneling to redox species in solution', J. Phys. Chem., Vol. 95, No. 2, pp. 877-886.

30 Amatore, C., Saveant, J.M., and Tessier, D. (1983) 'Charge-transfer at partially blocked surfaces - a model for the case of microscopic active and inactive sites', J. Electroanal. Chem., Vol. 147, No. 1-2, pp. 39-51. 